\title{
APPLICATION OF COMPUTATIONAL MECHANICS APPROACHES FOR INCREASING OF TRIBOSYSTEM OPERATIONAL PARAMETERS BY USING PLASMA HARDENING METHOD
}

\author{
Viacheslav Kopylov ${ }^{1}$, Oleg Kuzin ${ }^{1}$, Nickolai Kuzin ${ }^{2,3}$ \\ I National Technical University of Ukraine "Igor Sikorsky Kyiv Polytechnic Institute" \\ Kyiv, Ukraine \\ ${ }^{2}$ Lviv Research Institute for Forensic Examinations \\ Lviv, Ukraine \\ ${ }^{3}$ Lviv Branch of Dnipro National University of Railway Transport \\ Lviv, Ukraine \\ kopviacheslav@gmail.com,kuzin.oleg.an@gmail.com,kuzin.nick81@gmail.com
}

Received: 31 July 2020; Accepted: 26 January 2021

\begin{abstract}
On the basis of the mathematical model of the continual description of functional-gradient metal systems, taking into account their structure, a computational computer scheme has been developed that makes it possible to assess the stress-strain state of local volumes of parts taking into account their spatial inhomogeneity. With the use of modern software components of computational mechanics - FEniCS finite element analysis package and its implementation in Python, the optimal structural characteristics of wheelsets of railway locomotive tires after plasma treatment have been established. It is shown that, depending on the value of the load, hardening of products must be carried out to a depth of $4.5 \mathrm{~mm}$; with a further increase in thickness, the parameters of operational strength do not change.
\end{abstract}

MSC 2010: 74M15, 74S05, 74P10

Keywords: mathematical model, computational mechanics, contact problem, FEniCS, optimization

\section{Introduction}

Manufacturing of products with a specified life cycle based on computerization of all design and production stages is one of the most essential tasks of modern mechanical engineering. Its implementation is achievable through the joint use of methods of applied and computational mechanics when choosing rational technological modes of processing to obtain products with reliability parameters meeting operating conditions [1-3]. 
The application of new technologies and approaches of local gradient mechanics used to improve the service life of products is one of the features of modern mechanical engineering. It is a matter of introduction of components made of functionally gradient materials, which provide redistribution of a stress-strain state and temperature fields allowing essential changing of the design reliability [2, 3].

In this connection, a necessity of integration of new mathematical models of mechanics into computational schemes arises since they allow setting stress-strain state in local volumes of components taking into account their heterogeneity. This problem is especially important for the railway transport operation practice as friction knots determine the efficiency and reliability of the railway equipment operation by 80 to $90 \%$. Losses caused by wear and tear in the wheel-rail system range from 10 to $30 \%$ in trains used for traction $[4,5]$.

One of the advanced methods to address the problem of improving the tribosystem operational parameters are surface engineering technologies that allow control of the structural and energy state of external and internal surfaces of the critical parts interface in a fairly wide range $[2,3,6]$. The application of such technologies requires a scientific justification regarding the formation of an optimal distribution of nonlocal material characteristics in the surface component layers and should consider the specific features of power effects during the operation of structures.

To solve these significant scientific and practical issues, the mathematical apparatus of inverse problems from the domain of mathematics and mechanics, as well as computer modelling are involved, since in the majority of cases it is impossible to derive the solutions of such problems analytically.

\section{Statement of the problem of mechanics of increasing the contact strength of locomotive wheel tires using plasma hardening technologies}

Plasma hardening is one of the surface engineering technologies that changes the structural and energy state of material both in the surface and deep layers through the targeted thermal exposure. As a result, gradient structures are formed in the hardening zones. Their properties significantly differ from those zones that have not been treated.

Following hardening, an inhomogeneous structure is formed and its properties vary significantly in different zones. The highest values of hardness and microhardness are achieved on the contact surface, but the wear rate of this layer is substantially higher than that of the layer located at a depth of $1.5 \mathrm{~mm}$. This is conditioned by the formation of grain boundaries with different values of energy, the initiation of damages on the boundaries with its high level and the development of intercrystalline destruction. Local surface hardening zones hosting high angular boundaries with close grain boundary energy values are characterized by higher resistance to the formation of continuous damage, microscopic cracks and wear and tear $[6,7]$. 
At the level of mechanical properties, this leads to an increase in the value of elastic and structural characteristics in local volumes, which allows changing the contact durability of the parts in a wide range $[2,3,8]$.

However, the issues of formation of optimal distribution of structural material mechanical properties both in local volumes and as a whole, depending on external loads for achieving specified operational parameters of products, remain open.

Therefore, to solve the problem of providing the maximum increase of contact strength of a component at its functioning in conditions of prevailing stress-strain loads by optimizing the internal distribution of mechanical properties in local volumes of a material, we suggest the following mathematical formulation:

1. Let the studied body (component) occupy the spatial zone $X$, let us denote the body surface as $\partial X$.

2. Let us present the body surface as the collection of non-overlapping sets $\partial X=\partial X_{1} \cup \partial X_{2} \cup \partial X_{3}$, where $\partial X_{1}$ is the body area where loads are set, $\partial X_{2}$ is the body area with set displacement limits and $\partial X_{3}$ is the unloaded surface.

Regions $\partial X_{1}, \partial X_{2}, \partial X_{3}$ in turn can include simple subzones: $\partial X_{1}=\bigcup_{i=1}^{n} \partial X_{1}^{i}$, $\partial X_{2}=\cup_{j=1}^{m} \partial X_{2}^{i}, \partial X_{3}=\cup_{l=1}^{k} \partial X_{3}^{l}$.

3. The force vector $\vec{F}_{1}^{i}$ is set in each elementary subzone $\partial X_{1}^{i}$, and the displacement vector $\vec{u}_{2}^{j}$ is set in $\partial X_{2}^{i}$, which are distributed as follows in $\partial X$ : $\vec{F}_{1}^{i}=\vec{F}_{1}^{i}(x), \vec{u}_{2}^{j}=\vec{u}_{2}^{j}(x), x \in \partial X$.

4. When considering the mechanical properties of the studied component, we limit ourselves to such parameters as the elasticity modulus $E$, Poisson's ratio $\mu$ and the rupture resistance $\sigma^{*}$, the functional distribution of which in the zone $X$ corresponds to $E=E(x), \mu=\mu(x), \sigma^{*}=\sigma^{*}(x), x \in X$.

5. As a result, the stress state is formed in a component characterized in each point by a tensor $\hat{\sigma}=\hat{\sigma}(x), x \in X$.

6. As rated stress does not unambiguously define operational parameters of constructions [8], for the contact strength analysis we use such expression as:

$$
k(x)=1-\frac{\sigma_{m}(x)}{\sigma^{*}(x)}
$$

where $\sigma_{m}(x)$ is the equivalent representation of Mises's stress tensor, $\sigma^{*}(x)$ is material structural properties at a point. Let us name value $k(x)$ as the contact strength safety factor.

7. If $k(x)=1$, no softening is observed at the body point, if $k(x) \leq 0$, softening is observed at the body point.

8. The problem of increasing contact strength by optimizing the internal distribution of mechanical properties comes down to finding such functions as $E(x)$, 
$\mu(x), \sigma^{*}(x)$, which at set $\vec{u}_{i}(x)$ and $\vec{F}_{i}(x)$ ensure that the function (1) meets the condition (2):

$$
L=\frac{\operatorname{dim}_{x \in V}(k(x) \leq 0)}{\operatorname{dim}_{x \in V}(x)} \rightarrow \min ,
$$

where $\operatorname{dim}(\ldots)$ is the size of a zone that meets the specified condition. According to the relationship (2), the parameter $L$ is dimensionless.

\section{Solution of the problem regarding the optimization of the surface layer material structure to increase the contact strength of wheel set bandages}

To solve the problem described in section 2, we use approaches of computational mechanics, namely the FEniCS package of finite element analysis and its implementation in Python language [9]. This toolkit was chosen because of wide possibilities associated with the application of various mathematical models of the material continuum, including those built by the present authors, the availability of scaling function, adaptability for distributed computing, as well as the absence of any restrictions on its use for commercial, educational or scientific purposes.

Post-processing of finite element analysis results will be performed using the MatPlotLib freeware package for Python [10].

As a basic model for the material continuum, we used the model of elastic media taking into account damageability, offered in the work [11]. A basic equation of this model is the equation of continuum equilibrium at displacement:

$$
\left.+\frac{\vec{\nabla} \cdot\left(\frac{K(x)}{1-\sqrt{\alpha_{1}(\omega(x))^{2}+\alpha_{2}(|\vec{\nabla} \omega(x)|)^{2}+\alpha_{3}\left(\frac{1}{V_{0}} \int_{V} \omega d V\right)^{2}}}(\vec{\nabla} \cdot \vec{u}) \hat{I}+\right.}{\left.1-\sqrt{\alpha_{1}(\omega(x))^{2}+\alpha_{2}(|\vec{\nabla} \omega(x)|)^{2}+\alpha_{3}\left(\frac{1}{V_{0}} \int_{V} \omega d V\right)^{2}}\left(\vec{\nabla} \otimes \vec{u}-\frac{1}{3}(\vec{\nabla} \cdot \vec{u}) \hat{I}\right)\right)=0,}\right)
$$


where $\alpha_{1}, \alpha_{2}, \alpha_{3}$ are numerical constants, $\omega(x)$ is the damageability, $\vec{u}$ is the displacement vector, $\hat{I}$ is the ordinary tensor, $\vec{\nabla}$ is Hamilton's differential operator, $K(x)$ is the bulk modulus of elasticity, $G(x)$ is the shear modulus, $\otimes-$ tensor product, $\cdot$ is the scalar product, and $V_{0}$ is the material zone characteristic size at the mesoscale level equal to three grain diameters.

To solve the problem by the finite element method, we use the weighted residual method ("FEM weak formulation") [9], where as a weight function we choose vector $\vec{v}$ :

$$
\int_{\Omega}(\vec{\nabla} \cdot \hat{\sigma}) \cdot \vec{v} d x=0
$$

where $\Omega$ is the range of integration, $\hat{\sigma}$ is the stress tensor according to model (3), which is equal to

$$
\hat{\sigma}=\vec{\nabla} \cdot\left(\frac{K(x)}{1-\sqrt{\alpha_{1}(\omega(x))^{2}+\alpha_{2}(|\vec{\nabla} \omega(x)|)^{2}+\alpha_{3}\left(\frac{1}{V_{0}} \int_{V} \omega d V\right)^{2}}}(\vec{\nabla} \cdot \vec{u}) \hat{I}+\right.
$$

The essence of the "FEM weak formulation" consists in the selection of such a distribution of the desired function, which allows the basic condition - (4) to be provided as accurately as possible in a given functional space. The weak formulation is important tool that helps, in solving problems of FEM-mechanics, to pass from the continual description of mathematical equations to a system of linear algebraic equations, the solution of which is the discrete distribution of the desired quantity. In the case of solving equation (4), these are displacements, that are recalculated according to Hooke's law into stresses, and according to relation (1) into values of structural softening.

In this work, the values of the numerical constants $\alpha_{1}, \alpha_{2}, \alpha_{3}$ of the model (3) are taken equal to 0 to simplify the calculations.

Let us perform integration in respect of the parts of expression (4). Hence, we receive: 


$$
\int_{\Omega}(\vec{\nabla} \cdot \hat{\sigma}) \cdot \vec{v} d x=-\int_{\Omega} \hat{\sigma} \cdot \cdot(\vec{\nabla} \otimes \vec{v}) d x+\int_{\partial \Omega}(\hat{\sigma} \cdot \vec{n}) \cdot \vec{v} d s
$$

where $\vec{n}$ is the vector set at the body surface $\partial \Omega$.

Given the relation $\vec{T}=\hat{\sigma} \cdot \vec{n}$, where $\vec{T}$ is the surface vector, relation (5) is written as:

$$
\int_{\Omega}(\vec{\nabla} \cdot \hat{\sigma}) \cdot \vec{v} d x=-\int_{\Omega} \hat{\sigma} \cdot \cdot(\vec{\nabla} \otimes \vec{v}) d x+\int_{\partial \Omega} \vec{T} \cdot \vec{v} d s
$$

This equation is a basic one for solving the problem of finding operational parameters of structures working under friction conditions.

The problem is considered in a two-dimensional array. As an object of our research, we consider a two-dimensional rectangular body with functional-gradient depth properties, which lies on a rigid base. In the zone of contact interaction, let us set the parabolic distribution of the normal load component, the relationship between the normal and tangential components of the load vector is subject to the Coulomb's law:

$$
T_{2}(x)=\beta(x) T_{1}(x) \text {, }
$$

where $T_{1}(x)$ is a normal component of the load vector, $T_{2}(x)$ is tangential component of the load vector, $\beta(x)$ is a friction coefficient (Fig. 1).

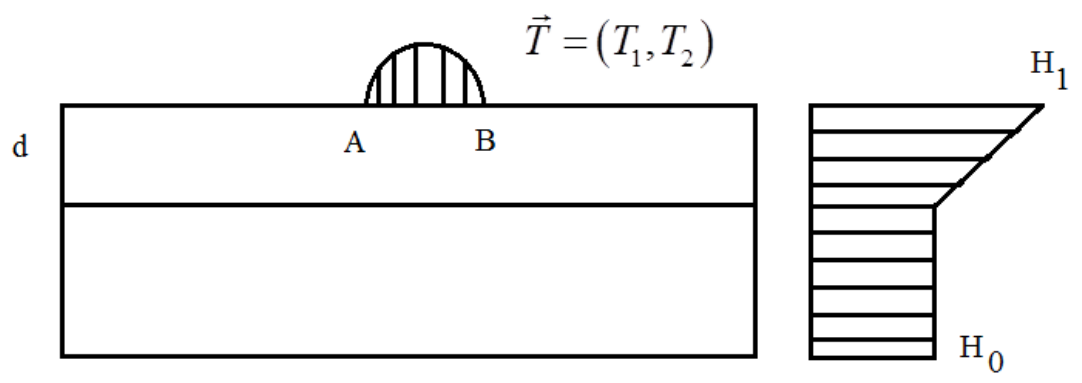

Fig. 1. The study object: $\mathrm{AB}$ is the contact patch (contact interaction area), $\vec{T}=\left(T_{1}, T_{2}\right)$ is the load vector, $d$ is the hardening zone thickness, $H_{1}$ is the surface microhardness value, $H_{0}$ is the micro-hardness value in the material depth

As the initial characteristic of distribution of material mechanical properties, we accept the microhardness value which characterizes the response of the element of a local volume with the given structural-energetic state to external influences. For transition from the microhardness value to the calculation of elastic and strength properties of materials, we use the results of the work [12].

It has been established that microhardness, measured by Vickers method, on the bandage surface of a locomotive wheel following plasma hardening is $H_{1}=3900 \mathrm{MPa}$, 
initial microhardness is $H_{0}=2600 \mathrm{MPa}$ detected at the depth of $d=2 \mathrm{~mm}$ from the surface, the initial stress-strain load is assumed to be equal $10 \cdot 10^{4} \mathrm{~N}$, and the friction factor $(\beta(x))$ is 0.25 [13].

Consequently, using the FEniCS package, the following stress distribution has been established in the studied structural fragment (Fig. 2).

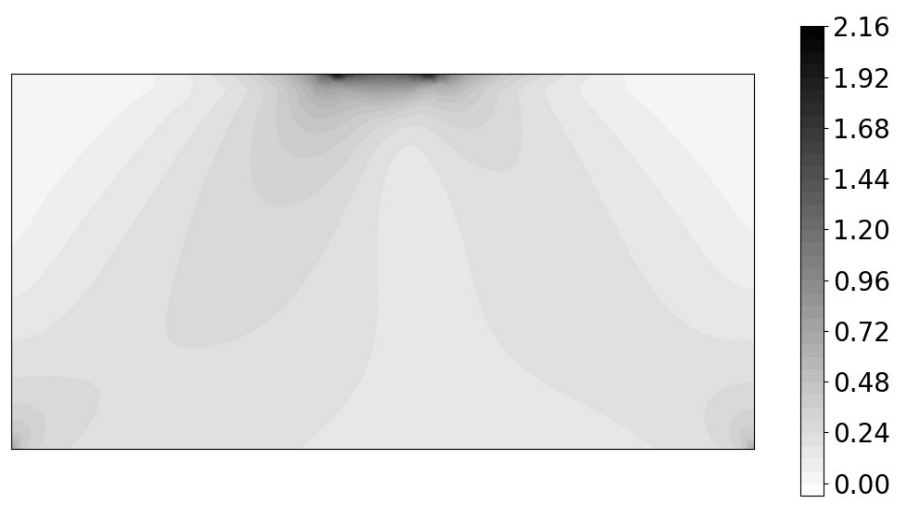

Fig. 2. Distribution of stress in the Mises form in the studied structure, $10^{9} \mathrm{~Pa}$

Analysis of the obtained results (Fig. 2) shows that the greatest value of stresses occurs in the immediate area of contact interaction. With distance from the contact zone, the stress values decrease, which makes it possible to suggest that behaviour of the surface zones in the contact load region are the most important for parts working under friction conditions.

Since the stress state does not unambiguously determine strength parameters of an object, we take structural softening according to the relation (2) as a characteristic of its operational properties (Fig. 3).

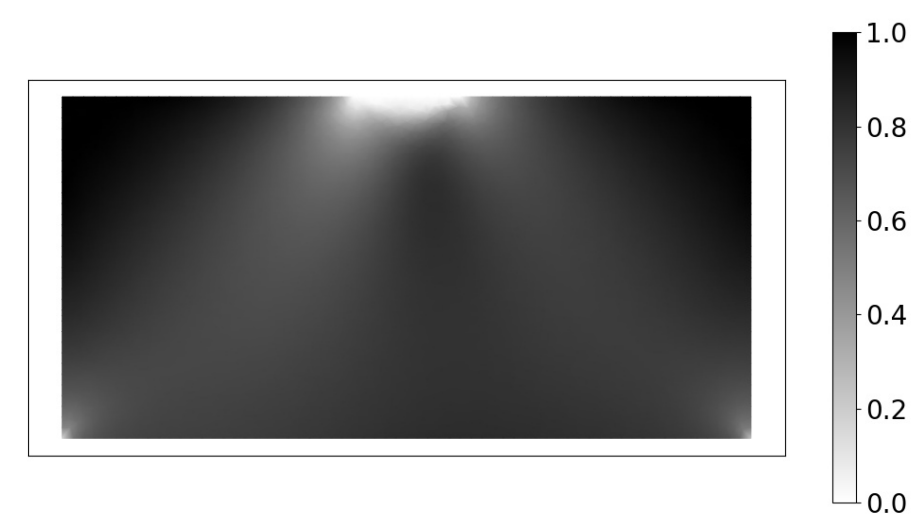

Fig. 3. Distribution of the structural softening value in a body

It was found that the greatest loss of strength is observed directly in the contact interaction zone (Fig. 3), which, with insufficient values of strength parameters, 
can be a source of crack initialisation. Structure optimization under various anticipated operating conditions, i.e. finding stress-strain distributions that provide the best performance, is an important aspect in studying the operation of the structure. We use the function (2) as an optimization criterion, and the thickness of hardened layer $d$ is considered as a control parameter.

To find the optimal modes of plasma hardening of wheelsets, the value of the size of the contact area $\mathrm{AB}$ will be considered equal to $13 \mathrm{~mm}$. Let us assume that the value of the vertical component of the load has the following discrete values $10 \cdot 10^{4} \mathrm{~N}, 12.5 \cdot 10^{4} \mathrm{~N}, 15 \cdot 10^{4} \mathrm{~N}$ (wheel load depends on the type of locomotive), and the value of the friction coefficient is discrete values $0.06,0.125,0.25,0.5$, which corresponds to the most common operating conditions of a real tribological wheel-rail system $[13,14]$. The value $d$ is measured in the range from 0 to $10 \mathrm{~mm}$.

As a result, the following dependence of the maximum softening zone on the parameter $d$ (Figs. 4-6) for different values of a normal load and friction coefficient can be obtained.

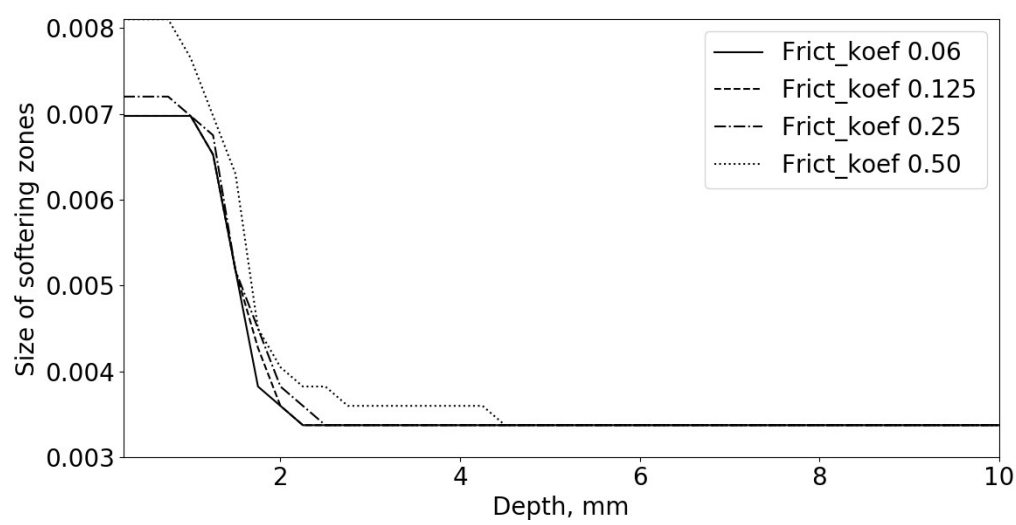

Fig. 4. Value of the softening zone for normal load $10 \cdot 10^{4} \mathrm{~N}$

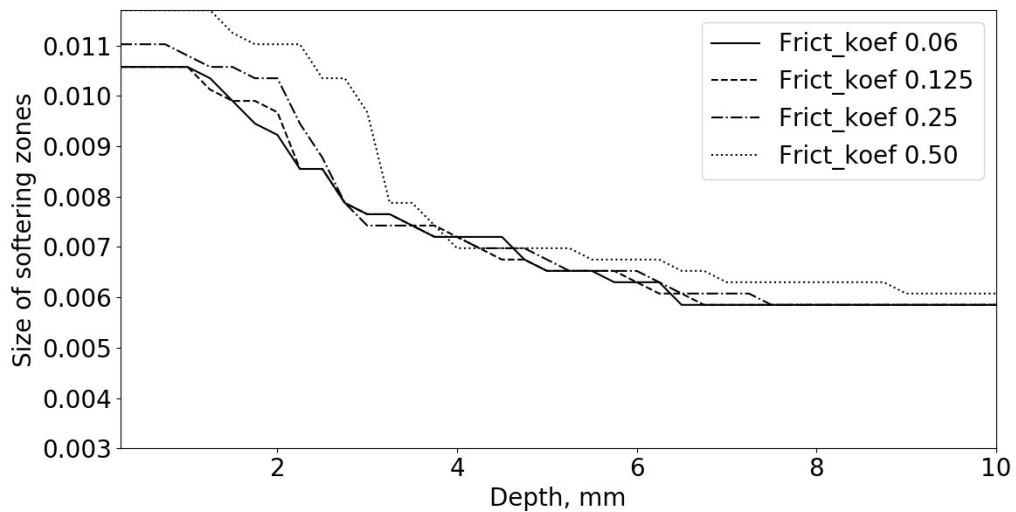

Fig. 5. Value of the softening zone for normal load $12.5 \cdot 10^{4} \mathrm{~N}$ 


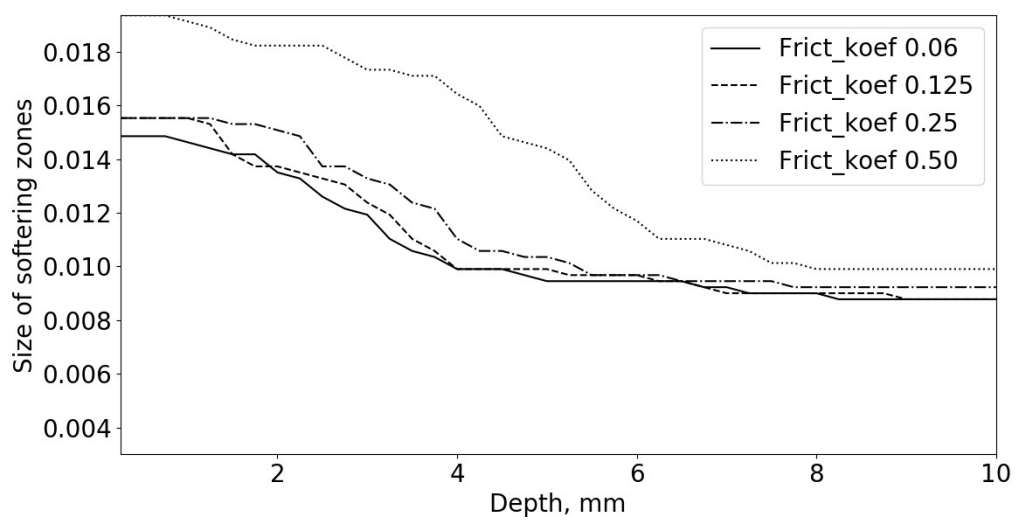

Fig. 6. Value of the softening zone for normal load $15 \cdot 10^{4} \mathrm{~N}$

As can be seen from the above calculations, there is a limit of the value of the hardened layer $d^{*}$, and, when this limit value is reached, the softening parameter does not decrease essentially.

At operating load $10 \cdot 10^{4} \mathrm{~N}$, depending on the friction coefficient, the parameter $d^{*}$ varies from 1.8 to $2.3 \mathrm{~mm}$, at $12.5 \cdot 10^{4} \mathrm{~N}$ it varies in the range from 2.8 to $3.3 \mathrm{~mm}$, and at $15 \cdot 10^{4} \mathrm{~N}$ - from 3.8 to $4.5 \mathrm{~mm}$. Further hardening is not feasible since at the same parameters of operational strength the energy loss for the surface treatment process increases.

In addition, an increase in softening parameter at friction factor growth is observed, which indicates changes in strength, parameters of wheels depending on specific features of the processes occurring during friction contact.

The developed approach makes it possible to choose plasma hardening modes to control the operational parameters of wheel sets in order to ensure their strength and durability.

\section{Conclusions}

1. Based on the approaches of computational and applied mechanics, the problem of optimization of operating parameters of locomotive wheel bandages following plasma strengthening has been considered.

2. The analysis of the structure stress state under proxy-operational loading modes using FEniCS FEM analysis package and its implementation in Python language has been carried out.

3. The necessity to harden wheel bandages to alternative depths depending on the operating conditions of a locomotive has been established. In this case, the increase of friction coefficient in the contact interaction zone from 0.06 to 0.5 does not require a significant change in the hardening layer thickness, and the increase in the normal component of friction load creates the need to increase the hardening layer thickness. 
4. It has been shown that the optimal thickness of bandage hardening layers should be selected depending on the operating conditions of a locomotive. For loads up to $10 \cdot 10^{4} \mathrm{~N}$, they should be hardened to the flange thickness up to $1.8 \ldots$ $2.3 \mathrm{~mm}$, at average load values of $12.5 \cdot 10^{4} \mathrm{~N}-$ up to $2.8 \ldots 3.3 \mathrm{~mm}$, and at $15 \cdot 10^{4} \mathrm{~N}$ and more - up to $3.8 \ldots 4.5 \mathrm{~mm}$.

5. Hardening of locomotive wheels bandages to the thickness of more than $4.5 \mathrm{~mm}$ is not feasible, since at the same parameters of operational strength the energy costs for the technological process are increased.

\section{References}

[1] Kukla, S., \& Siedlecka, U. (2020). Time-fractional heat conduction in a finite composite cylinder with heat source. Journal of Applied Mathematics and Computational Mechanics, 19(2), 85-94, DOI: 10.17512/jamcm.2020.2.07.

[2] Lyashenko, B. (2010). Optimization of Coating Technology in Terms of Strength and Wear Resistance. Kiev: Institute for Strength Problems (in Russian).

[3] Dwivedi, D.K. (2018). Surface engineering enhancing life of tribological components. Springer, DOI: $10.1007 / 978-81-322-3779-2$.

[4] Drozdov, Ju. (2010). Applied Tribology (Friction, Wear, Lubrication). Moscow: Eco-Press (in Russian).

[5] Iwnicki, S., Spiryagin, M., Cole, C., \& McSweeney, T. (2020). Handbook of Railway Vehicle Dynamics. CRS Press, DOI: 10.1201/9780429469398.

[6] Louisette, P. (2013). Grain Boundaries. From Theory to Engineering. Springer, DOI: 10.1007/978-94-007-4969-6.

[7] Kopylov, V., Kuzin, O., \& Kuzin, N. (2019). Improving contact durability of polycrystalline systems by controlling the parameters of large-angle grain boundaries. Eastern-European Journal of Enterprise Technologies, 5, 12(101), 14-22, DOI: 10.15587/1729-4061.2019.181441.

[8] Troshenko, V. (2005). Strength of Materials and Structures. Kiev: Akademperiodika (in Russian).

[9] Langtangen, N., \& Logg, A. (2016). Solving PDEs in Python. The FEniCS Tutorial I. Springer Open, DOI: 10.1007/978-3-319-52462-7.

[10] Johansson, R. (2019). Numerical Python: Scientific Computing and Data Science Applications with Numpy, SciPy and Matplotlib. Apress. DOI: 10.1007/978-1-4842-4246-9.

[11] Kuzin, O., Lukiaynets, B., \& Kuzin, N. (2019). Continual description of polycrystalline taking into account their structure. Technology Audit and Production Reserves, 1/1(45), DOI: $10.15587 / 2312-8372.2019 .156159$.

[12] Bulychev, S.N. (1999). The ratio between recovered and unrecovered hardness when tested by nanomicroindentation. Technical Physics Journal, 69, 7 (in Russian).

[13] Guidelines to best practices for heavy haul railway operations: wheel and rail interface issues (2001). International Neavy Naul Association.

[14] Keropyan, A.M., Verzhansky, P.M., Mostakov, V.A., \& Basov, R.K. (2016). Dependence of the coefficient of friction in the contact zone of the wheel-rail system on the roughness of the interacting surfaces. Mining Information and Analytical Bulletin, 11 (in Russian). 